\title{
NDC80 promotes proliferation and metastasis of colon cancer cells
}

\author{
X.K. Xing ${ }^{1}$, H.Y. Wu ${ }^{2}$, H.L. Chen ${ }^{1}$ and H.G. Feng ${ }^{1}$ \\ ${ }^{1}$ Department of Life Science and Technology, Xinxiang Medical University, \\ Xinxiang, China \\ ${ }^{2}$ Department of Forensic Biology, Xinxiang, China \\ Corresponding author: X.K. Xing \\ E-mail: biyingxiao@163.com
}

Genet. Mol. Res. 15 (2): gmr.15028312

Received December 21, 2015

Accepted February 26, 2016

Published May 6, 2016

DOI http://dx.doi.org/10.4238/gmr.15028312

\begin{abstract}
Chromosome instability is a common feature of tumor cells, and may be an important mechanism in tumor formation. Nuclear division cycle 80 (NDC80) is closely associated with the stability of chromosomes. Therefore, we investigated the relationship between NDC80 and development of colon cancer using a range of methods. Westernblotting and immunohistochemistry were employed to determine the expression of this protein in different colon cells and tissues, cell proliferation was measured with an MTT assay, levels of proliferating cell nuclear antigen were examined by immunofluorescence, and cell migration was observed using wound healing tests. Our results showed that the expression of NDC80 in colon cancer cells (CACO2, HCT8, HCT116, and SW480) and tissues (from 20 patients) was higher than that in controls. Moreover, cell proliferation and migration rates were elevated in cells transfected with NDC80 compared to control groups. In summary, NDC 80 promotes the proliferation and metastasis of colon cancer cells, and may constitute a new target for gene therapy in treating
\end{abstract}


this disease. Combined with clinicopathological grading, measurement of positive NDC80 expression may be helpful in diagnosing and estimating the prognosis of colon cancer patients.

Key words: Chromosome instability; Colon cancer; Hec1; Metastasis; Nuclear division cycle 80; Proliferation

\section{INTRODUCTION}

Colon cancer is a common malignancy of the digestive tract, usually occurring in the rectum and its junction with the sigmoid colon. It is one of the most common malignant tumors in western Europe, North America, and other developed countries (Zou et al., 2014). Owing to its high recurrence rate, it represents a serious public health problem (Bishnupuri et al., 2010). At present, treatment is principally based on surgery and radiotherapy. This approach is effective for patients in the early stages of this disease, but achieves poor outcomes for those with advanced cancer and microcarcinoma. With the development of molecular biology and related sciences, gene targeting therapy has become a research topic of significant interest in tumor treatment (Zhang et al., 2014; Maus et al., 2015). Gene targeting relies on the selection of a gene for treatment, therefore, the identification of a specific target affecting tumors is important.

Chromosome instability is a common feature of tumor cells, and may be an important mechanism in tumor formation (Venkitaraman, 2001; Luo et al., 2011; Venkitaraman, 2014). Accurate regulation of the binding and dynamic changes of microtubules is essential for chromosome stability. The kinetochore is a complex of multiple proteins that plays a key role in this process. Nuclear division cycle 80 (NDC80), known in humans as highly expressed in cancer $(\mathrm{Hec} 1)$, is a nuclear protein rich in coiled-coil motifs. It was discovered by Durfee et al. (1993) using yeast two-hybrid technology and the C-terminus of the retinoblastoma protein (RB) to screen a B lymphocyte complementary DNA library. NDC80 complexes are important to the spindle assembly checkpoint, and participate in the regulation of mitosis (Ciferri et al., 2008; Alushin et al., 2012). In this study, we analyzed the biological function of NDC80 in the proliferation and metastasis of colon cancer cells, and provided new reference data and experimental support for colon cancer-targeting gene therapy.

\section{MATERIAL AND METHODS}

\section{Materials}

Normal human intestinal epithelial NCM460 cells and the colon cancer cell lines SW480, HCT8, HCT116, and CACO2 were purchased from the Chinese Academy of Medical Sciences tumor hospital. Between January 2015 and November 2015, 20 colon cancer patients (42-58 years old, twelve men and eight women) were recruited from the First Affiliated Hospital of Xinxiang Medical College, and underwent surgical resection of tumorous and adjacent tissue. Pathological examination showed that of these, seven cases were highly differentiated adenocarcinoma, six were moderately differentiated adenocarcinoma, and seven were undifferentiated carcinoma. Twelve cases demonstrated lymph node metastasis, while eight did not. Six patients were at stage I, five at stage II, five at stage III, and four at stage IV (according to the tumor-nodemetastasis staging of colon cancer). All patients gave their written informed consent. 


\section{Main reagents}

Fetal bovine serum (FBS) was purchased from Sijiqing Hangzhou Biological Engineering Materials Company (Hangzhou, China), Dulbecco's modified Eagle's medium (DMEM) and trypsin were sourced from Gibco (Waltham, MA, USA), Lipofectamine was provided by Invitrogen (Carlsbad, CA, USA), mouse anti-human NDC80 and rabbit anti-human $\beta$-actin antibodies were purchased from Chemicon (Billerica, MA, USA), mouse anti-human proliferating cell nuclear antigen (PCNA) antibody was manufactured by Beijing Jinqiao Biotechnology (Beijing, China), and enhanced chemiluminescence reagent was produced by Pierce (Waltham, MA, USA). An ECLIPSE 80i fluorescence microscope was also used (Nikon, Tokyo, Japan).

\section{Detection of NDC80 protein expression in different cells by western blot}

Normal human intestinal epithelial NCM460 cells, and colon cancer SW480, HCT8, HCT116, and CACO2 cells were cultured in DMEM containing $10 \% \mathrm{FBS}$ at $37^{\circ} \mathrm{C}$ in a $5 \% \mathrm{CO}_{2}$ atmosphere. Total protein was extracted and separated by sodium dodecyl sulfate-polyacrylamide gel electrophoresis, before being transferred to a cellulose nitrate membrane. Skim milk powder was used to block the membrane for $1 \mathrm{~h}$ at $37^{\circ} \mathrm{C}$. Anti-NDC80 antibody was then added and incubated overnight with shaking at $4^{\circ} \mathrm{C}$. The membrane was then exposed to horseradish peroxidase-labeled secondary antibody for $1 \mathrm{~h}$ at $37^{\circ} \mathrm{C}$. Bound antibodies were visualized by chemiluminescence, and images were scanned with a Bio-Rad ChemiDoc MP (Hercules, CA, USA) gel imaging system to obtain gray values using the Quantity One software.

\section{Detection of NDC80 protein expression in different colon tissues by immunohistochemistry}

Healthy and cancerous paraffin-embedded colon tissue was cut into 5-mm slices and immunohistochemically stained. The expression of NDC80 was observed under a microscope.

\section{Gene transfection}

Experiments comprised control, empty plasmid, and NDC80-transfected groups. When SW480 cells reached 60 to $70 \%$ confluence, the serum volume fraction of the medium was reduced to $5 \%$. Lipofectamine $(100 \mu \mathrm{L})$ was then mixed with $1 \mu \mathrm{g}$ plasmid for $20 \mathrm{~min}$, and added to the culture medium. After $6 \mathrm{~h}$, this was replaced with medium containing $10 \%$ serum, and $48 \mathrm{~h}$ later, culture medium containing $200 \mathrm{ng} / \mathrm{L}$ G418 was used to select transfected cells. After two weeks, 24-well plates were used to clone and amplify selected cells.

\section{3-(4,5-Dimethylthiazol-2-yl)-2,5-diphenyltetrazolium bromide (MTT) assay for measurement of cell proliferation}

Cell density was adjusted to $2 \times 10^{4} / \mathrm{mL}$ and $100 \mu \mathrm{L}$ of cells per well was added to a 96 -well plate, with six wells for each experimental group. MTT reagent $(20 \mu \mathrm{L} /$ well $)$ was added to cell cultures of various ages (1-6 days), and incubated for $4 \mathrm{~h}$ at $37^{\circ} \mathrm{C}$ in a $\mathrm{CO}_{2}$ incubator. The culture medium was then carefully removed and $150 \mu \mathrm{L}$ dimethyl sulfoxide added to wells, before shaking the plate for $10 \mathrm{~min}$. Absorbance at $570 \mathrm{~nm}$ was measured in each well using a microplate reader, and a cell growth curve was generated. 


\section{PCNA expression detected by immunofluorescence assay}

Cell were fixed for 30 min with $4 \%$ formaldehyde at room temperature, then incubated with phosphate-buffered saline (PBS) containing 1\% Triton X-100, before being blocked with goat serum for $30 \mathrm{~min}$. After removal of the serum, PCNA antibody was incubated with cells at $4^{\circ} \mathrm{C}$ overnight. Cells were then exposed to fluorescein isothiocyanate (FITC)-labeled secondary antibody at $37^{\circ} \mathrm{C}$ for $45 \mathrm{~min}$. After being washed with PBS and mounted, cells were observed under a fluorescence microscope. PCNA expression was determined as follows: PCNA-positive rate $=$ FITC-positive cells/total cells x 100\%. Twenty fields of view were examined each sample.

\section{Wound healing}

When cell confluence approached $80 \%$, a $10-\mu \mathrm{L}$ plastic pipette tip was used to scratch the culture gently, and then pictures were taken every $12 \mathrm{~h}$ under a microscope. Cultures were observed for $72 \mathrm{~h}$, and the width of the scratch was measured.

\section{Statistical analysis}

Data were analyzed by the $t$-test using SPSS 13.0 (SPSS Inc., Chicago, IL, USA), and $P$ values $<0.05$ were considered statistically significant.

\section{RESULTS}

\section{NDC80 protein expression in different colon cells}

NDC80 protein was highly expressed in CACO2, HCT8, HCT116, and SW480 colon cancer cells, but levels in normal human intestinal epithelial NCM460 cells were significantly decreased in comparison (Figure 1).

\section{HCT8 HCT116 CACO2 SW480 NCM460}

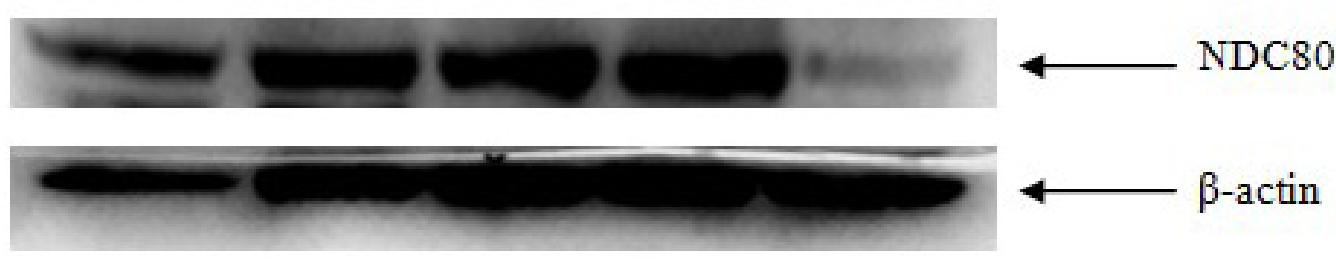

Figure 1. Expression of NDC80 protein in various colon cells measured by western blot.

\section{NDC80 protein expression in different colon tissues}

NDC80-positive tissues were identified by yellow or brownish yellow staining. Of the 20 samples of normal colonic tissue, seven $(35 \%)$ were positive, while among the 20 colon cancer specimens, $15(75 \%)$ were positive. This difference was statistically significant $(\mathrm{P}<$ 0.05 ; Figure 2). 
A

B

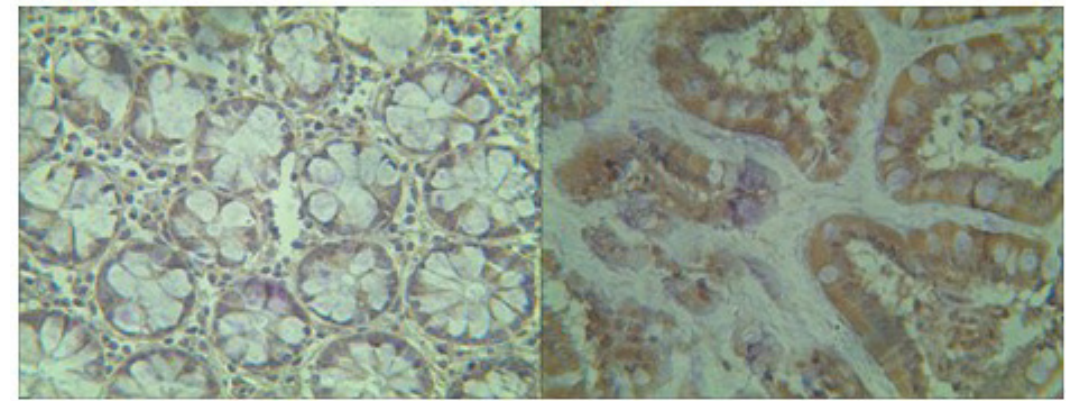

Figure 2. Expression of NDC80 protein in different colon tissues visualized by immunohistochemical staining (200X magnification). A. Normal colon tissue; B. colon carcinoma.

\section{MTT-based cell growth curve}

Proliferation of SW480 cells in the NDC80-transfected group was greater than that of the control groups at the same time points, and this difference was statistically significant (P $<0.05$ ). The growth rate of SW480 cells did not significantly differ between the control and empty plasmid groups $(\mathrm{P}>0.05$; Figure 3$)$.

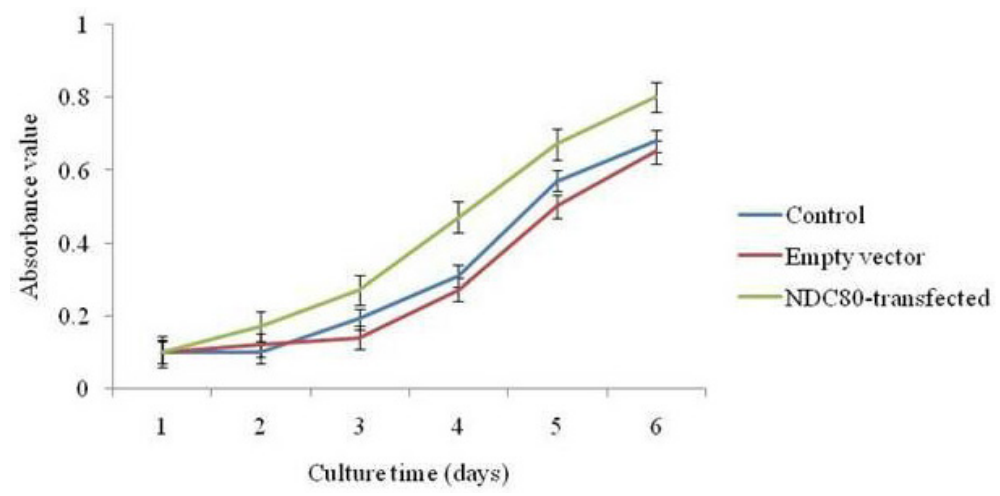

Figure 3. Growth curves of cells in each group.

\section{Cellular PCNA expression}

PCNA-positive rates in the control, empty plasmid, and NDC80-transfected groups were $24.2 \pm 3.4,30.4 \pm 5.6$, and $45.1 \pm 5.5 \%$, respectively. Compared to the control and empty plasmid groups, PCNA expression in the NDC80-transfected group was significantly higher $(\mathrm{P}<0.05$, Figure 4).

\section{SW480 cell migration}

$72 \mathrm{~h}$ later,the widths of the wounds created in cultures of SW480 cells in the control, empty plasmid, and NDC80-transfected groups were $89.27 \pm 6.23,80.34 \pm 7.16$, and $64.77 \pm$ 
$9.18 \mu \mathrm{m}$, respectively. SW480 cells in the $N D C 80$-transfected group gradually migrated to the middle of the wound, shortening the distance between the two sides. In the control and empty vector groups, there were significantly fewer SW480 cells in the wound area, and the distance between the two sides was greater than that observed with the NDC80-transfected group, to a statistically significant degree $(\mathrm{P}<0.05$, Figure 5$)$.
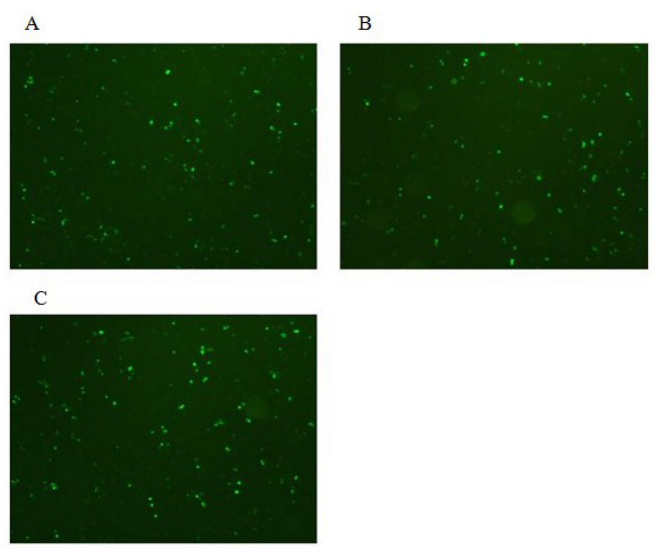

Figure 4. PCNA expression in each group was visualized by immunofluorescent staining (100X magnification). A. control group, B. empty plasmid group, C. NDC80-transfected group.
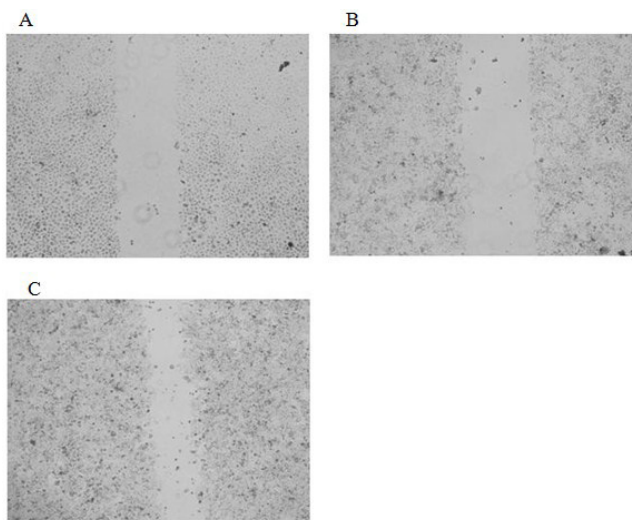

Figure 5. Wound healing in each group (100X magnification). A. control group, B. empty plasmid group, C. NDC80-transfected group.

\section{CONCLUSIONS}

NDC80 is a nuclear protein with a coiled-coil structure, consisting of 642 amino acids. Its $\mathrm{N}$-terminus contains at least three functional binding sites: a predicted nuclear localization sequence, a Nek2 phosphorylation site, and a specific RB interaction site. The C-terminus incorporates a series of seven leucine heptad repeat sequences, which play an important role in protein interactions (DeLuca et al., 2005). NDC80 is highly conserved in eukaryotic cells. 
NDC80 is a cell cycle protein expressed during cell division. In particular, levels of this protein significantly increase from $\mathrm{S}$ to $\mathrm{M}$ phase, and it has a regulatory function during the G2/M phase (Sundin et al., 2011). NDC80 expression in all cells undergoing division is high, and it is not expressed in terminally differentiated cells. This protein maintains chromosome stability (Wei et al., 2011; Wei et al., 2014), and in cells with abnormal NDC80 expression, spindle checkpoint dysfunction, abnormal chromosome separation, and cell cycle disorder may occur, perhaps ultimately leading to tumor formation (Alushin et al., 2012; Tang and Toda, 2015). NDC80 assembly plays an important role in the spindle checkpoint process.

Nuclear aneuploidy results from unstable chromosomes, and is a common feature of tumor cells. A previous study found that aspects of tumor behavior, such as recurrence and invasion, correlate with the number of aneuploid cells. The development of aneuploidy is an important mechanism in tumor development. (Shinmura et al., 2014). Colon cancer is a common malignant tumor of the digestive system associated with development, lifestyle, genetic factors, and colorectal adenoma. Cytogenetic analysis has revealed most colon cancers to be aneuploid. Many variations of chromosome number exist, including sub-diploidy, pseudodiploidy, and tetraploidy (Baker and van Deursen, 2010). The distribution of aneuploidy in a tumor is an important indicator of progression and invasion; treatment effectiveness and prognosis for aneuploid tumors are significantly worse than those seen in relation to diploid tumors.

This study found that NDC80 protein was highly expressed in SW480, HCT8, HCT116, and $\mathrm{CACO} 2$ colon cancer cells, and the NDC80-positive rate was significantly higher in colon cancer specimens than in normal colon tissues. Moreover, MTT and PCNA assays revealed faster cell proliferation in the NDC80-transfected group, while wound healing tests showed greater migration ability in cells transfected with NDC80 compared to controls. Our results demonstrate that NDC80 is significantly associated with invasion and metastasis in colon cancer. This protein may promote the occurrence of aneuploidy, leading to the development of this disease. NDC80 may therefore represent a new target for colon cancer gene therapy. The results of this study provide a new theoretical basis for gene therapy in colorectal cancer. However, the number of samples in our study was limited, and the role of NDC80 in colon cancer development was examined using only in vitro experiments, limiting the strength of any conclusions drawn. In future work, in vivo tests involving expanded sample sizes will be carried out to verify the present results.

\section{Conflicts of interest}

The authors declare no conflict of interest.

\section{ACKNOWLEDGMENTS}

Research supported by research grants from the Key Research Project of Henan Province (\#15A180020) and the National Natural Science Foundation of China (\#U1304819, 81401519).

\section{REFERENCES}

\footnotetext{
Alushin GM, Musinipally V, Matson D, Tooley J, et al. (2012). Multimodal microtubule binding by the Ndc80 kinetochore complex. Nat. Struct. Mol. Biol. 19: 1161-1167. http://dx.doi.org/10.1038/nsmb.2411

Baker DJ and van Deursen JM (2010). Chromosome missegregation causes colon cancer by APC loss of heterozygosity. Cell Cycle 9: 1711-1716.http://dx.doi.org/10.4161/cc.9.9.11314
} 
Bishnupuri KS, Luo Q, Sainathan SK, Kikuchi K, et al. (2010). Reg IV regulates normal intestinal and colorectal cancer cell susceptibility to radiation-induced apoptosis. Gastroenterology 138: 616-626, 626.e1-626.e2. http://dx.doi. org/10.1053/j.gastro.2009.10.050

Ciferri C, Pasqualato S, Screpanti E, Varetti G, et al. (2008). Implications for kinetochore-microtubule attachment from the structure of an engineered Ndc80 complex. Cell 133: 427-439. http://dx.doi.org/10.1016/j.cell.2008.03.020

DeLuca JG, Dong Y, Hergert P, Strauss J, et al. (2005). Hec1 and nuf2 are core components of the kinetochore outer plate essential for organizing microtubule attachment sites. Mol. Biol. Cell 16: 519-531. http://dx.doi.org/10.1091/mbc. E04-09-0852

Durfee T, Becherer K, Chen PL, Yeh SH, et al. (1993). The retinoblastoma protein associates with the protein phosphatase type 1 catalytic subunit. Genes Dev. 7: 555-569. http://dx.doi.org/10.1101/gad.7.4.555

Luo F, Poulogiannis G, Ye H, Hamoudi R, et al. (2011). Mutant K-ras promotes carcinogen-induced murine colorectal tumourigenesis, but does not alter tumour chromosome stability. J. Pathol. 223: 390-399. http://dx.doi.org/10.1002/ path. 2790

Maus MK, Hanna DL, Stephens CL, Astrow SH, et al. (2015). Distinct gene expression profiles of proximal and distal colorectal cancer: implications for cytotoxic and targeted therapy. Pharmacogenomics J. 15: 354-362. http://dx.doi. org $/ 10.1038 /$ tpj.2014.73

Shinmura K, Kurabe N, Goto M, Yamada H, et al. (2014). PLK4 overexpression and its effect on centrosome regulation and chromosome stability in human gastric cancer. Mol. Biol. Rep. 41: 6635-6644.http://dx.doi.org/10.1007/s11033014-3546-2

Sundin LJ, Guimaraes GJ and Deluca JG (2011). The NDC80 complex proteins Nuf2 and Hec1 make distinct contributions to kinetochore-microtubule attachment in mitosis. Mol. Biol. Cell 22: 759-768. http://dx.doi.org/10.1091/mbc.E10$\underline{08-0671}$

Tang NH and Toda T (2015). MAPping the Ndc80 loop in cancer: A possible link between Ndc80/Hec1 overproduction and cancer formation. BioEssays 37: 248-256. http://dx.doi.org/10.1002/bies.201400175

Venkitaraman AR (2001). Chromosome stability, DNA recombination and the BRCA2 tumour suppressor. Curr. Opin. Cell Biol. 13: 338-343. http://dx.doi.org/10.1016/S0955-0674(00)00217-9

Venkitaraman AR (2014). Tumour suppressor mechanisms in the control of chromosome stability: insights from BRCA2. Mol. Cells 37: 95-99. http://dx.doi.org/10.14348/molcells.2014.2346

Wei R, Ngo B, Wu G and Lee WH (2011). Phosphorylation of the Ndc80 complex protein, HEC1, by Nek2 kinase modulates chromosome alignment and signaling of the spindle assembly checkpoint. Mol. Biol. Cell 22: 3584-3594. http://dx.doi.org/10.1091/mbc.E11-01-0012

Wei X, Gao C, Luo J, Zhang W, et al. (2014). Hec1 inhibition alters spindle morphology and chromosome alignment in porcine oocytes. Mol. Biol. Rep. 41: 5089-5095. http://dx.doi.org/10.1007/s11033-014-3374-4

Zhang D, Huang Y and Wang H (2014). [Advances of driver gene and targeted therapy of non-small cell lung cancer]. Zhongguo Fei Ai Za Zhi 17: 750-754.

Zou AM, Wang HF, Zhu WF, Wang FX, et al. (2014). Effect of RNAi-mediated silencing of Livin gene on biological properties of colon cancer cell line LoVo. Genet. Mol. Res. 13: 3832-3841.http://dx.doi.org/10.4238/2014.May.16.8 